\title{
Medical Image of the Week: Central Venous Access with Dextrocardia
}

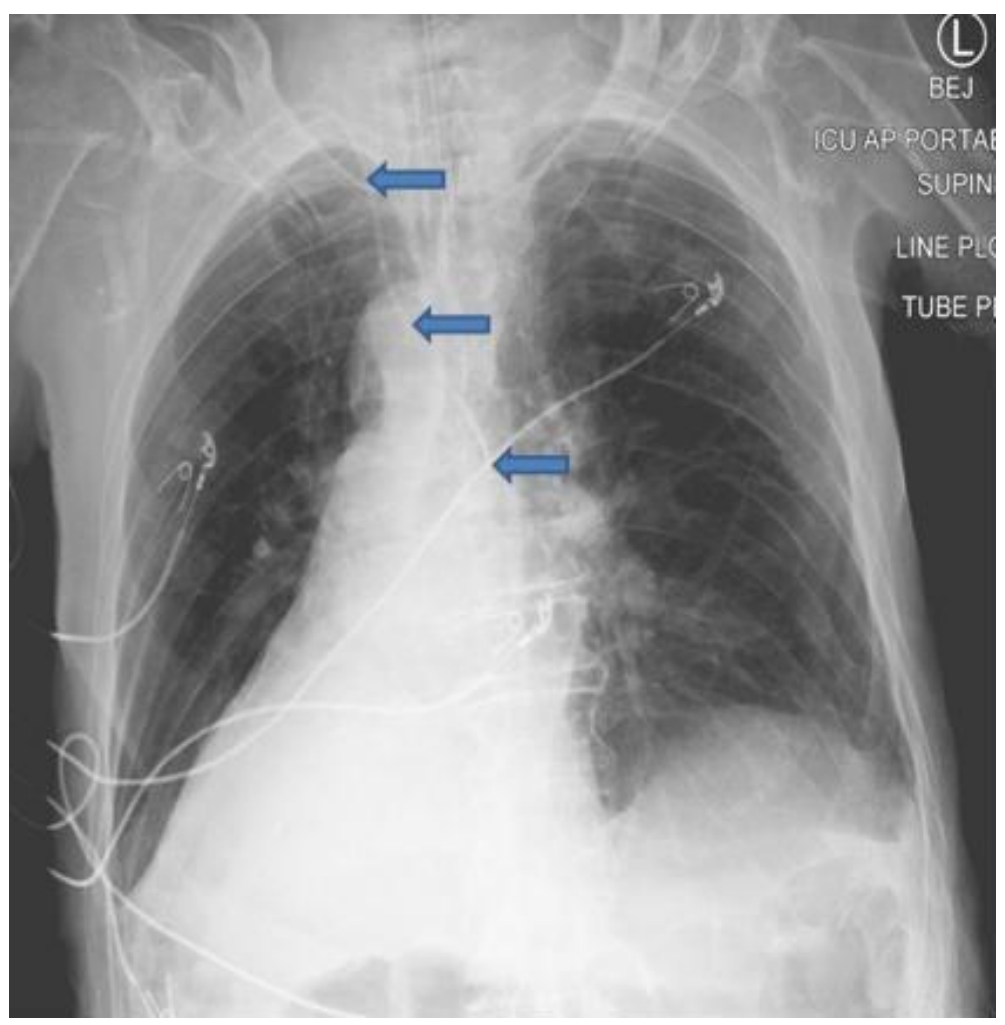

Figure 1. Post CVC placement chest X-ray. Catheter traced with arrows.

An 88-year old man, with known dextrocardia, was admitted with a diagnosis of septic shock. A right sided internal jugular central venous catheter was placed uneventfully using ultrasound guidance. Chest X-ray obtained after the catheter placement is shown (Figure 1). Although the utility of a chest $X$-ray after every ultrasound guided central line placement is questionable, it continues to be "routine practice" in many centers. In dextrocardia, a right sided central line is expected to cross the midline as in this patient. When in doubt, the catheter may not be used unless venous placement is confirmed.

Venous placement of the catheter can be confirmed by:

1. Transducing the catheter and confirming venous waveform;

2. Blood gas analysis consistent with venous gas;

3. Imaging X-ray or cross sectional (1).

Bhupinder Natt MD

Division of Pulmonary, Allergy, Critical Care and Sleep

Banner-University Medical Center, Tucson, AZ USA

\section{Reference}

1. Morton PG. Arterial puncture during central venous catheter insertion. Crit Care Med. 1999 May;27(5):878-9. [CrossRef] [PubMed] 